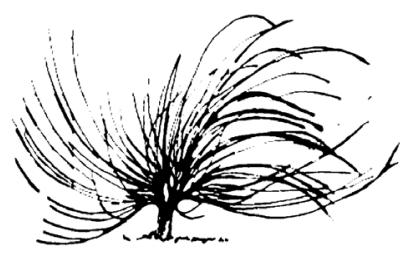

\title{
Factores que dificultan el aprendizaje de la filosofía en los estudiantes universitarios
}

\author{
Gabriela Rangel Díaz ${ }^{1}$ \\ Universidad Teológica de América Central \\ Costa Rica \\ gabrielarange102@gmail.com
}

\begin{abstract}
Resumen
Este trabajo investigativo tiene por objeto identificar los factores que dificultan el aprendizaje de la filosofía de los estudiantes de la Universidad Teológica de América Central (UTAC). El estudio se realizó con la participación de dieciocho estudiantes en su mayoría extranjeros, y siete docentes de la Escuela de Filosofía, se efectuaron dos grupos focales con el alumnado y entrevistas en profundidad con el profesorado. Todos los discentes fueron hombres pertenecientes a diversas congregaciones religiosas. Los resultados presentan los principales factores culturales, de comprensión lectora, de pérdida de cursos, por sistemas educativos de procedencia, pedagógicos y personales que dificultan el aprendizaje de la filosofía desde los aportes del profesorado y del estudiantado.

Palabras clave: dificultad en el aprendizaje, educación superior, estudiante universitario, filosofía
\end{abstract}

\begin{abstract}
This investigative work aims at identifying the factors that hinder Philosophy learning in students from Universidad
\end{abstract}

Recibido: 18 de julio de 2019. Aprobado: 29 de abril de 2020.

http://dx.doi.org/10.15359/rep.15-2.6

1 Docente de Filosofía en la Escuela de Estudios Generales de la Universidad de Costa Rica y de la Escuela de Filosofía de la Universidad Teológica de América Central. Magíster en Educación con énfasis en Pedagogía Universitaria de la Universidad Nacional de Costa Rica. https://orcid.org/0000-0002-1635-5864 
Teológica de América Central (UTAC). This study was conducted with the participation of eighteen students (mostly foreign) and seven faculty from the School of Philosophy; two focus groups with the students were done, and in-depth interviews were carried out with the faculty. All the students were male belonging to different religious congregations. The findings show the main cultural, reading comprehension, course failure, educational system provenance, pedagogical, and personal factors that hinder Philosophy learning from the perspective of both university faculty and students.

Keywords: Learning difficulties, university students, higher education, Philosophy

\section{Introducción}

$\mathrm{E}$

sta investigación sistematiza los principales hallazgos de una experiencia de trabajo de campo que se inició durante el primer semestre de 2015 en la Universidad Teológica de América Central (UTAC) con la participación del cuerpo docente de la Escuela de Filosofía compuesto por siete profesores y una muestra de dieciocho estudiantes inscritos en varios cursos de la carrera.

Esta institución nace desde los años 70 como el Instituto Teológico de América Central (ITAC). Sin embargo, es hasta el año 2011 que se transforma en la Universidad Teológica de América Central Monseñor Óscar Arnulfo Romero (UTAC). La población estudiantil que atiende es $98 \%$ extranjera, masculina y pertenece a diversas órdenes religiosas como los Dominicos, Calasancios, Capuchinos, Trinitarios, Agustinos, Jesuitas, Combonianos, Amigonianos, Franciscanos, etc. En su mayoría los discentes provienen de diferentes latitudes como lo son: El Salvador, Haití, Panamá, Nicaragua, Honduras, México, Colombia y Costa Rica, entre otras.

La estancia en Costa Rica de estos educandos se debe a que las congregaciones a las que pertenecen, los envían para iniciar sus estudios de teología y filosofía, por considerar que en Costa Rica el cuerpo docente de filosofía está altamente capacitado y que la oferta de asignaturas es más amplia que en otros países. Sin embargo, uno de los problemas que se presentan en la UTAC se relaciona con los niveles de promoción de los estudiantes en los cursos de filosofía; los registros de calificaciones muestran que es normal que estos discentes 
presenten exámenes extraordinarios en las materias de filosofía, debido a que no logran aprobarlas en el periodo ordinario. Muchos de ellos reprueban al menos una de dichas asignaturas y ha habido casos aislados en los que los estudiantes han llegado a perder todo el bloque de materias en un semestre.

Los y las docentes de la UTAC han manifestado reiteradamente que las dificultades de los alumnos extranjeros para aprender filosofía se deben a deficiencias de su formación preuniversitaria, pues al momento de ingresar a la educación superior no han logrado adquirir ciertas habilidades básicas como: la comprensión lectora, el razonamiento deductivo e inductivo, la construcción de hipótesis o enunciados, la capacidad para dar ejemplos y contraejemplos o argumentar e inferir ideas, lo que se refleja en sus producciones escritas, pero también en la producción oral.

Debido a lo anteriormente explicado, el objetivo de este estudio fue identificar los factores que dificultan el aprendizaje de la filosofía de los estudiantes de la Universidad Teológica de América Central según su país de procedencia.

La experiencia investigativa inició desde enero del 2015 y finalizó en julio del 2016 mediante reuniones con el estudiantado y el profesorado de modo que se generó una participación voluntaria, consciente y responsable durante el periodo que cubrió el estudio. Este se llevó a cabo mediante el uso de instrumentos como grupos focales y entrevistas en profundidad con los alumnos y los docentes respectivamente.

La importancia de sistematizar esta información recae en que es la primera investigación en el nivel nacional que recopila las percepciones de una muestra de estudiantes universitarios, en su mayoría extranjeros, respecto a las dificultades en el aprendizaje de la filosofía que han tenido que afrontar desde un contexto educativo muy específico en Costa Rica.

\section{Antecedentes}

\section{El abordaje pedagógico de la filosofía}

Los estudios que se han realizado en Costa Rica para analizar la problemática en el aprendizaje de la filosofía, muestran que existen situaciones muy particulares que afectan el adecuado desarrollo de los procesos educativos en esta doctrina desde etapas finales del colegio hasta la universidad. Para Gómez (2011), la filosofía es una disciplina 
que se aprende mediante la acción de filosofar "y solo se aprenden asuntos filosóficos cuando se hace filosofía sobre ellos" (p. 124). Gómez (2011) critica el desinterés del sistema educativo costarricense al incluirla en el currículo de educación secundaria hasta el último año de estudios y, además, que algunas temáticas propias de esta área del conocimiento se aborden desde asignaturas ajenas a ella, tal es el caso de la ética y la lógica, que suelen desarrollarse en las lecciones de religión y de español, respectivamente. En cuanto a las clases de filosofía, Gómez (2011) señala que, en muchos casos, se limitan a "la reproducción acrítica de conocimientos y datos históricos, de moralizaciones y de academicismo" (p. 127), lo que claramente no fomenta espacios para la reflexión filosófica y el desarrollo de la criticidad.

El trabajo de Carvajal y García (2004) muestra que el enfoque historicista también está presente en las clases de filosofía en el nivel universitario. Su estudio consideró una muestra de 390 estudiantes que cursaban el primer año de estudios generales en la Universidad Nacional (UNA), la Universidad de Costa Rica (UCR) y el Instituto Tecnológico de Costa Rica (TEC). La mitad de los participantes indicaron haber tenido malas experiencias con los cursos de esta asignatura debido a tres razones: 1) el curso en sí, 2) el profesor y 3) las actividades.

Algunas de las causas más relevantes que identificaron los alumnos para justificar su visión negativa hacia la filosofía son las siguientes:

Acerca del curso: dicen que no hubo análisis y era muy histórico (Carvajal y García, 2004)

Acerca del profesor: aducen que los docentes no intentan aumentar la capacidad de razonamiento de sus estudiantes. Adicionalmente mencionaron que uno de sus mentores no era profesor de filosofía, sino de psicología (Carvajal y García, 2004).

Sobre las actividades: dicen que son clases aburridas en las que el tiempo es mal aprovechado, por cuanto se hablaba más de la vida de los filósofos que de su pensamiento. Los alumnos debían apegarse a textos que no se explicaban en clase (Carvajal y García, 2004).

Según Carvajal y García (2004), en la actualidad son pocos los y las estudiantes que conciben una relación entre la filosofía y la realidad, aun cuando el $72 \%$ de los 390 alumnos indicaron que la filosofía les presenta una nueva visión de mundo que promueve la reflexión crítica 
y el análisis. Los investigadores indican que estas apreciaciones varían según el curso, el docente y la universidad. Aclaran, además, que "los estudiantes prefieren cursos con temas de actualidad en donde predomine el análisis crítico y reflexivo, en los que se promueva la participación del estudiante. También, exigen que los métodos de enseñanza filosófica sean dinámicos y flexibles" (Carvajal y García, 2004, p.16). En sus conclusiones insisten en revisar y analizar el proceso pedagógico que se utiliza para enseñar y aprender filosofía.

\section{La preparación académica previa de los estudiantes universitarios}

Los estudios que se han realizado en otras instituciones de educación superior enLatinoamérica para analizar la problemática en el aprendizaje de la filosofía, muestran que existen falencias académicas desde antes de incorporarse a la universidad, al respecto Difabio (2015) señala que el estudiantado que ingresa a la formación superior no tiene una adecuada preparación para asumir la exigencia intelectual que plantea la universidad. Su trabajo pone énfasis en aspectos metacognitivos para evaluar si los y las participantes eran capaces de construir procesos y estrategias implicados en la comprensión de textos y del pensamiento crítico. El estudio se llevó a cabo en la Universidad Nacional de Cuyo con 20 participantes (un hombre y diecinueve mujeres), dos docentes, seis psicopedagogas y el resto estudiantes universitarias de las carreras de Formación Docente, Psicopedagogía, Ciencias de la Educación y Profesorado de Filosofía. Sus edades oscilaban entre 21 y 35 años, con una media de 25. En la investigación se empleó la prueba de comprensión lectora CLOZE. Dentro de las conclusiones más relevantes se señala que la comprensión lectora se ve afectada por tres variables: el lector, el texto y la instrucción. En los sujetos participantes se comprobó que los factores que inciden en la eficiencia de esta capacidad son el conocimiento previo, la memoria de trabajo y la metacomprensión.

El estudio de Difabio (2015) dictaminó que:

Se ha comprobado el elevado porcentaje de estudiantes del ciclo superior de la escuela media y de los primeros cursos de Universidad ( $30 \%$ o más) tienen dificultades para resolver eficazmente problemas que exigen un pensamiento abstracto y, por tanto, insuficientemente equipados para hacer frente al desafío intelectual de la enseñanza universitaria. (p. 343) 
Durante los años 2007 y 2008, Calderón y Quijano (2010) utilizaron la prueba CLOZE para identificar las características y habilidades de comprensión lectora de una muestra de estudiantes de derecho y de psicología de la Universidad Cooperativa de Colombia seccional Barrancabermeja. Los investigadores concluyeron que los y las participantes presentaban un alto nivel de frustración, al percatarse de que no poseen un manejo instrumental efectivo de la competencia lingüística, es decir, no muestran un manejo gramatical del lenguaje, ni de la sintaxis, la fonética y la semántica, dentro de las micro y macro estructuras de un texto. Según estos estudiosos colombianos "entender un asunto y no comprender otro es construir un camino que no conduce sino a la confusión pero no a dilucidar el mensaje del texto mismo" (Calderón y Quijano, 2010, p. 339).

Gutiérrez y Montes de Oca (2007) llevaron a cabo un estudio en la Universidad Juárez Autónoma de Tabasco para dar a conocer cuál era la situación de las habilidades lectoras de los estudiantes en esa región de México. Según Gutiérrez y Montes de Oca (2007) el mayor acceso tecnológico que se tiene en la actualidad genera una mayor exposición de las personas a la información, lo que exige agudizar su capacidad de discriminar e interpretar los símbolos, pero esa capacidad está muy disminuida o no ha sido bien desarrollada. Concluyen que lo anterior se debe a que la estructura de la educación tradicional y los programas que emplean, están muy lejos de las necesidades actuales, se trabaja la comprensión lectora como un simple proceso de decodificación, sin profundizar en la construcción activa de conocimientos.

Echeverría (2004) considera que el aprendizaje de la filosofía forma en habilidades de pensamiento tales como: contrastar, detectar similitudes, dar buenas razones, inferir visiones de mundo, pensar hipotéticamente, proponer ejemplos y contra-ejemplos, autocorregirse, descubrir contradicciones, entre otras. Sin embargo, estas habilidades de pensamiento solo se desarrollan gracias a la lectura y a la discusión de lo leído. En la etapa universitaria, el intercambio de ideas y el debate de las mismas es fundamental para alcanzar un dominio efectivo de las habilidades de pensamiento. 


\section{Marco teórico}

\section{Dificultades en el aprendizaje de la filosofía desde latitudes lejanas}

La UNESCO (2011) precisa que en países de África las dificultades en el aprendizaje de la filosofía se ligan a dos circunstancias: en primer lugar, a la formación de los y las profesionales, y en segundo lugar a la fuerte ausencia de esta asignatura en secundaria en países anglófonos, a lo mucho se conserva como materia optativa, similar situación se da en Reino Unido y en Estados Unidos. Por tanto, si los discentes han tenido nulo o escaso contacto con la materia de filosofía y los procesos de comprensión que esta implica, entonces su adquisición se torna más dificultosa.

En Marruecos, por ejemplo, se ha identificado que los y las estudiantes que pasan del colegio a una formación superior, presentan más dificultades para responder a "la exigencia de pasar progresivamente de un modelo pedagógico centrado en la participación activa de los y las estudiantes en la clase" (UNESCO, 2011, p. 66), a un modelo que demanda una lectura directa de los textos y elaborar una amplia discusión en torno a ellos, como lo demanda la materia de filosofía.

El aprendizaje de la filosofía demanda un posicionamiento desde diferentes perspectivas de pensamiento, sin embargo, la UNESCO (2011) señala que tradicionalmente la formación que se le ha brindado al estudiantado en esta disciplina ha sido mayoritariamente desde la perspectiva de la filosofía occidental, propiamente europea, de modo que no se han integrado otras tradiciones de pensamiento como la oriental o la latinoamericana. La formación filosófica que se brinda en el nivel secundario y universitario se basa en manuales y textos de autores de Occidente, lo que omite el pensamiento local. Lo precedente dificulta que los estudiantes se apropien del conocimiento filosófico cuando lo visualizan como totalmente descontextualizado de sus realidades y por ende sin utilidad.

En países como la "República Centroafricana, hay un curso de filosofía africana en el que se estudian autores africanos desde un enfoque comparativo con los autores occidentales" (UNESCO, 2011, p. 52), en Costa Rica, todavía no se ha dado ese paso, la enseñanza continúa siendo mayoritariamente con las ideas de los filósofos europeos occidentales, situación contraria a la que sucede en Jamaica en donde desde la universidad se brinda formación filosófica a partir de los autores locales. 
En la enseñanza de la filosofía en Malí se estableció que el plurilingüismo puede ser un problema en el aprendizaje de esta disciplina. Dado que según el profesor Touré esas dificultades en el aprendizaje "están estrechamente vinculadas a la lengua de enseñanza" (UNESCO, 2011, p. 81). Esto porque el sistema educativo de este país africano es consecuencia de la colonización y este proceso también ha conllevado que las alumnas y los alumnos de este territorio deban aprender una lengua extranjera que no es la que usan cotidianamente para expresarse: el francés. Situación homologable se da en Haití, país cuya historia ha sido marcada con el fierro de la colonia, al presente existen dos idiomas oficiales, el más usado por la población es el idioma criollo haitiano, en esa lengua se da la educación y según estudios de León (2007) se estima que en Haití solo el $10 \%$ de la población sabe hablar francés, segunda lengua oficial, más propia de los sectores cultos en la ciudad y a su vez lengua minoritaria junto con el español.

Vygotsky (1995) señala que en el ambiente no solo hay leyes naturales, sino que están determinadas social e históricamente. El desarrollo del pensamiento está condicionado por: los instrumentos lingüísticos del pensamiento, el lenguaje y la experiencia sociocultural. En coherencia con lo anterior, el texto de Okoudjou (2007) “¿Cómo enseñar hoy la filosofía en África?" según se cita en la UNESCO (2011), declara que: "aprender a pensar y a escribir en la lengua materna crea todas las oportunidades para un advenimiento de una filosofía africana entendida al singular como al plural ya que una vez más, la filosofía se encuentra en la lengua y en la cultura maternas" (p. 81). Por tanto, el lenguaje y el idioma determinan las categorías conceptuales con las que entendemos el mundo y accedemos al conocimiento.

\section{Dificultades en el aprendizaje de la filosofía en Latinoamérica}

El profesor Sergio Espitaleta Hoyos a través de una investigación que realizó en 1993 para la Universidad de Antioquia, identificó que los y las estudiantes de filosofía presentaban una serie de dificultades para el aprendizaje de esta disciplina, entre ellas enumera las siguientes:

1. El estudiante nunca ha tenido contacto, ingreso, ni inducción a la filosofía, lo que lo hace llegar a ella, en su primer curso de la media vocacional, con prenociones y expectativas que no son bien orientadas y aprovechadas por el profesor. 
2. La filosofía se ocupa, entre otros asuntos, de la reflexión y los alcances del ser, del conocer, del pensar, y del hacer, sin embargo, el estudiante no posee los instrumentos necesarios para entender dicha reflexión porque nunca se le ha fomentado a su manera, en cursos previos, una disposición al análisis, a la síntesis, a la emisión de juicios o de opiniones $\mathrm{y}$, menos aún, a la lectura, escritura y sus implicaciones.

3. La filosofía presenta, en general, un panorama aparentemente árido para el estudiante que, jamás se ha encaminado por ella y sus senderos, como la abstracción, la lógica y el raciocinio, el juicio, la inducción, la deducción, etc.,

4. Hay problemas en la transmisión y en la recepción de la filosofía que se manifiesta tanto en asimilación, transferencia y operacionalización de conceptos, como en el abandono de todo esfuerzo que represente generar pensamiento o razonamiento formal e hipotético o, al menos, habilidad analítica y sintética. (Espitaleta, 1993, p.103)

Este especialista enfatiza que la enseñanza de la filosofía ha sido tradicionalmente imponer saberes y visiones de mundo, sin incluir al otro que puede brindar otras formas de saber y de mundo.

Un elemento que puede dificultar el aprendizaje de la filosofía en el cuerpo estudiantil es que los sistemas educativos no han penetrado en las dimensiones afectivas, exploratorias e imaginativas de los y las discentes. Luego, no se suelen tomar en cuenta los intereses de las alumnas y los alumnos, ni su experiencia previa. Considera Espitaleta (1993) que la enseñanza de la filosofía debe romper con los esquemas rígidos que aún se conservan, cambiar los métodos de enseñanza por otros que faciliten el intercambio y la mutua construcción de conocimiento entre discentes y docentes, lo que genera pensamiento formal y lateral.

La filosofía debe verse como un acto creador, al respecto Rengifo y Pinillo (2013), consideran que es un espacio en dónde todo filósofo se avoca a esa labor y todo educador y educadora enseña al alumno y la alumna a desaprender. La filosofía como proceso educativo es un acto de liberación, de desaprensión. No obstante, estos investigadores colombianos convergen en que uno de los problemas de la enseñanza y aprendizaje de la filosofía es que "la acción de filosofar ha sido reducida a un radio de acción muy corto y fugaz, el de las elucubraciones 
conceptuales, las argumentaciones extensas y la repetición constante de un pensamiento erudito" (p. 2).

La lectura y el análisis de las ideas en filosofía han perdido protagonismo debido a que actualmente las y los educandos se manejan en el mundo del internet, las imágenes y lo audiovisual, esto ya forma parte de su imaginario colectivo, individual y de sus intereses, restándole importancia a la lectura y a la escritura en la forma tradicional, al punto de que

...el papel de la lectura y la escritura de talante argumentativa, propositiva, interpretativa, liberadora, genérica, creativa y crítica, pasen a ser consideradas, por los educandos, conocimientos de escaza importancia, en tanto que conciben estos saberes como destrezas innecesarias para enfrentarse al mundo mediato cibernético. (Rengifo y Pinillo, 2013, p. 5)

El aprendizaje de la filosofía es una vivencia personal, pero el y la docente debe hacer lo posible por incluir los intereses afectivos y cognitivos de los y las estudiantes, a fin de que puedan sentir empatía por la materia e irse apropiando de sus conceptos. Además de eso es clave un correcto planteamiento de lo que se desea enseñar, pues mejora el grado de aprendizaje que alcanzarán los alumnos y las alumnas. Asimismo, el material que se utilice debe ser significativo para el y la discente, que despierte su curiosidad, organizado científicamente y apoyado en una evaluación no solamente sumativa, sino sobre todo formativa. Una estrategia que puede estimular lo anterior, consiste en dar mayor profundidad en el tiempo destinado a la discusión y el análisis filosófico. Carvajal y García (2004) señalan que los y las docentes en la educación diversificada estudian más los autores clásicos que otras áreas, es hasta la universidad que la o el educando tiene más libertad para expresarse y construir sus propias ideas. Es decir, ese ejercicio de analizar, discutir y expresar las ideas debiera ser un imperativo desde el colegio, para que al ingresar a la universidad los discentes perfeccionen más esas habilidades y se apropien del conocimiento filosófico con total libertad del manejo de sus ideas y la comprensión de las mismas.

La enseñanza por parte del cuerpo docente y el aprendizaje por parte de las y los educandos debe: 
...estar inclinada a la indagación y formulación de estrategias socioafectivas y cognoscitivas que faciliten no sólo la transmisión y la recepción de conocimientos sobre la misma, sino que permita que el estudiante desarrolle habilidades de pensamiento que lo potencien para enfrentar el reto del mundo actual que exige niveles de racionalidad alta y creciente. (Espitaleta, 1993, p. 110)

Lo recomendable es favorecer estrategias de aprendizaje inscritas desde un ambiente de esfuerzo y alegría, que promuevan el trabajo individual y grupal. Se espera que el y la estudiante durante la secundaria alcance niveles de razonamiento formal, hipotético y operacional, entre otras habilidades de pensamiento que debieran estar afianzadas para cuando ingresan a la universidad.

\section{La comprensión lectora de los universitarios}

En la actualidad, la población estudiantil que ingresa a las universidades es hija de la tecnología, pertenece a la generación de los millennials, los cuales construyen su mundo desde la imagen que aportan los dispositivos electrónicos y ejercen más la lectura de textos en versiones digitales que impresas. No obstante, todavía quedan jóvenes que disfrutan también los libros en papel.

En ambos casos, la comprensión lectora implica procesamiento de información y diversas habilidades que debieran aprenderse progresivamente desde las primeras etapas formativas. Calderón y Quijano (2010) puntualizan que la mayor dificultad para los y las discentes respecto a la comprensión lectora deriva de que "la mayoría no sabe leer, no comprende lo que lee porque no ha tenido las bases fundamentales para un buen proceso literal; otros están relacionados con la manera como proyectan sus propios pre-saberes en la lectura del texto" (Calderón y Quijano, 2010, p. 360).

La comprensión lectora en el nivel universitario demanda el dominio de competencias lingüísticas como: el manejo gramatical del lenguaje, de la sintaxis, de la fonética y de la semántica, así como saber distinguir las micro y macro estructuras de un texto. Todas las características anteriores son deseables en los estudiantes que cursan la carrera de filosofía. Sin embargo, al presente los docentes se confrontan con que el estudiante promedio se enfoca más en la comprensión literal de un texto, y no es capaz de profundizar en la metacomprensión, es decir, 
ir más allá y construir inferencias. Además, las generaciones actuales que ingresan a la educación superior, lo hacen con un léxico muy reducido y con incapacidad de leer entre líneas. Desde las teorías de Claux y La Rosa (2004), la lectura es un ejercicio constructivo sumamente complejo, ya que implica procesos de interacción entre las características del lector o lectora y las del texto, además de relaciones de significado que se generan de parte del objeto y del sujeto.

En el presente el tema de la comprensión lectora en la universidad genera confrontaciones entre los profesores, quienes se quejan de que los estudiantes no leen y estos últimos a su vez de que les dejan muchas lecturas. La lectura es una herramienta para la comprensión de las ideas, Calderón y Quijano (2010) señalan que el cuerpo docente se ha percatado de los problemas de comprensión lectora a través de errores frecuentes de comprensión en los análisis de documentos escritos, ensayos, trabajos de investigación y otros que presentan los estudiantes. Por tanto, la comprensión lectora es un ejercicio complejo para el cual los discentes universitarios no siempre están preparados.

En coherencia con lo anterior Amador y Alarcón (2006) consideran que el problema del estudiantado universitario es concreto: el bajo nivel de prácticas lectoras, y los escasos hábitos para la lectura. Esto se puede comprender mejor desde el libro La teoría de las seis lecturas de Miguel de Zubiría (1995), según se cita en Calderón y Quijano (2010),

...la lectura ocupa uno de los últimos lugares en la lista de las cosas que hacen felices a los ciudadanos y cómo el hablar de lectura puede parecer un contrasentido, a pesar las campañas institucionales de promoción de la lectura. Leer es algo que no le gusta a la gente, a pesar de la publicidad que despliegan las editoriales monopólicas cuando publican sus best-seller; leer tiene cada vez menos demanda. (p. 340)

Este es uno de los principales factores que aquejan a la población colombiana universitaria. La falta de interés por la lectura se desarrolla mucho antes de ingresar a la universidad lo que condiciona el desarrollo de habilidades lectoras.

Lo precedente es una situación que no sucede solo en Colombia, sino también en países como México. Según Gutiérrez y Montes de 
Oca (2007), la lectura es un gran problema en el contexto universitario, debido a que en general entre los y las estudiantes mexicanos se dan

escasos o nulos hábitos de lectura, dificultades para comprender y analizar textos, pobre información acerca de los acontecimientos locales, nacionales y mundiales, desconocimiento de métodos de lectura y estudio, poco interés hacia la investigación bibliográfica y documental, mala ortografía y desconocimiento de los principios básicos de redacción. (p. 10)

Los efectos de las insuficiencias mencionadas se reflejan en diversos problemas académicos como en el desarrollo de las investigaciones y trabajos, baja participación en los cursos, apropiación de un vocabulario limitado o escaso, por ende menor dominio de la expresión oral y escrita, escaso aprovechamiento de los sistemas de bibliotecas y sus beneficios. A lo anterior se suma una gran desmotivación hacia la lectura, al valorarla de forma negativa como una obligación. Estos estudiantes también son sujetos de la cultura fotocopia o copilación, que consiste en folletos con escasas referencias bibliográficas, esto impide que el o la discente se interesen por consultar libros originales en las bibliotecas.

Aunado a lo anterior, el tiempo que se le consagran a la lectura los discentes en la educación superior suele ser reducido "un $48.4 \%$ de los universitarios mexicanos dedica entre una y cinco horas a la semana a la lectura de textos escolares" (Gutiérrez y Montes de Oca, 2007, p. 7). Siendo esto insuficiente, pues según estos investigadores mexicanos un o una estudiante universitario promedio debiera dedicar 13 horas por semana a la lectura para poder responder a los cursos inscritos desde el primer año y en coherencia con la cantidad de créditos matriculados.

Las generaciones actuales poseen mayor acceso a libros e investigaciones impresas y en línea. Sin embargo, llama la atención por un lado que los y las estudiantes universitarias se limitan a visitar las bibliotecas de sus facultades, rara vez se aventuran a otras bibliotecas fuera del campo de la entidad de educación superior. Y por otro lado, la cultura fotocopia debido a que "el $86.3 \%$ de los estudiantes universitarios de nuestro país sacan fotocopias para cumplir con la obligación de leer y hacer sus tareas e investigaciones académicas, mientras el $40.7 \%$ declaró que utilizan frecuentemente Internet para satisfacer sus necesidades de información" (Gutiérrez y Montes de Oca, 2007, p. 8). 
Además el $11 \%$ de los y las estudiantes universitarios compran libros o revistas, y $46.4 \%$ no compran nada para mejorar sus investigaciones (Gutiérrez y Montes de Oca, 2007). Es decir, la preocupación por leer, actualizarse y mejorar los conocimientos en filosofía y otras disciplinas de formación universitaria, no es una prioridad para el alumnado. Aun cuando la lectura ha venido tomando gran importancia en el ámbito educativo y cultural, sigue habiendo un enorme rezago y resistencia a desarrollar la lectura en la educación superior, tanto en países desarrollados como subdesarrollados.

\section{Carencias de lecto-escritura de los estudiantes de filosofía en Amé- rica Latina}

Los estudiantes universitarios presentan carencias de lectoescritura, que están de manera directa asociadas con sus experiencias lectoras y escriturales. Al respecto Velásquez (2002) enfatiza que la carencia de lectoescritura para acceder a los textos filosóficos es una de las principales dificultades para el aprendizaje de la filosofía. Para comprender la causa de esas falencias es necesario remitirse al sistema educativo mismo en el cual el educando es formado. En América Latina, predomina mucho la tradición oral como forma de adquirir cultura, "De ahí que cuando a los estudiantes se les asignan lecturas como trabajos de clase, arrugan el ceño o se sienten molestos, porque para ellos la lectura simboliza tedio" (Velásquez, 2002, p. 54).

Este comportamiento no es único de los y las estudiantes, también está presente en los profesores y las profesoras. En Colombia, las estadísticas sobre hábitos de lectura y los procesos de lectoescritura son muy bajos, mientras que en los países desarrollados una persona se lee quince libros al año o más, en este país del sur solo uno (Velásquez, 2002). La lectura y la escritura no es un asunto solo de eficacia, sobre la cantidad de libros que se lee al año, sin embargo, la lectura está ligada al desarrollo de ciertas habilidades de pensamiento propias de la filosofía como el goce estético, la capacidad de pensar por sí y abrirse a otras formas de pensamiento.

Leer y escribir no son meramente instrumentos al servicio de presentar exámenes o trabajos, sino que implican ejercicios de pensamiento crítico, de discusión, de tomar posturas y de discernir frente al documento que se estudia. Por tanto, es necesario que el y la docente 
motiven al cuerpo estudiantil de filosofía a leer y luego a escribir pequeños ensayos, para mejorar sus habilidades en lectoescritura.

Arrieta y Meza (2005), destacan que los y las estudiantes de universidad tienen problemas de escritura, debido a carencias o vacíos en la comprensión lectora. Consecuencia de lo anterior también es el analfabetismo funcional que consiste en la imposibilidad de identificar las ideas principales en un texto y la intención del autor, así como redactar de manera incoherente. Las deficiencias en la lectura y la redacción se deben a

...un desfase entre los conocimientos que trae el estudiante de Educación Media y Diversificada y el grado de exigencia propio del nivel de Educación Superior. No corresponde al sistema de Educación Superior la tarea de enseñar a los estudiantes a leer y a escribir; sería lógico presumir que ellos dominan estas destrezas lingüísticas, pero los datos obtenidos en esta investigación nos demuestran lo contrario. (p. 2)

Por tanto, se puede afirmar que las dificultades para la lectura que presentan los estudiantes universitarios residen en gran medida en falencias propias de la etapa de secundaria. No obstante, el problema más grande consiste en que aún existe un gran desconocimiento por parte de las y los estudiantes de sus propias deficiencias de lectura, no suelen interesarse con profundidad en investigar sobre el vocabulario que desconocen cuando leen, y menos en el uso del diccionario para textos que son más especializados. La filosofía como disciplina demanda el manejo de un lenguaje específico que solo se adquiere poco a poco con la lectura, el estudio y experiencias de comprensión.

La lectura, la escritura y el diálogo son herramientas que favorecen un correcto aprendizaje de esta disciplina. Actualmente, falta promoverlas más en los cursos de filosofía. Además de flexibilizar más los currículos de las asignaturas e introducir pensadores locales.

\section{Metodología}

El enfoque de la investigación fue cualitativo debido a que el análisis de los datos se realizó desde una concepción fenomenológica e inductiva. El objetivo fue comprender los comportamientos humanos desde el propio marco de referencia de quien actúa. 
Esta pesquisa se llevó a cabo bajo el paradigma naturalista-humanista o interpretativo, que consiste según Barrantes (2013) en examinar los significados de las acciones humanas y de la vida social. Desde este, se hace un esfuerzo por comprender el objeto de estudio inserto en las reacciones humanas y en la experiencia del ser humano, no solo como individuo sino también como totalidad contenida en un contexto determinado.

El propósito de la indagación fue identificar los factores que dificultan el aprendizaje de la filosofía en los estudiantes universitarios según país de procedencia. Para estos efectos, se trabajó con el cuerpo docente de la Escuela de Filosofía de la Universidad Teológica de América Central (UTAC) compuesto por siete profesores y con una muestra de 18 estudiantes universitarios que estaban inscritos en los cursos de la carrera de filosofía. En el estudio los docentes participaron a través de entrevistas en profundidad y los discentes mediante grupos focales. Ambas técnicas se aplicaron con un consentimiento informado, cuyas normas éticas estipulaban: 1) un compromiso de confidencialidad de la información, 2) el anonimato de los participantes y 3) la no la difusión de los resultados hasta que el último estudiante se retirara del país para no afectar su proceso en Costa Rica.

Asimismo, con el objetivo de mejorar la aproximación conceptual, se recurrió a la consulta de más de cincuenta fuentes bibliográficas para apoyar las temáticas incluidas en el estudio, dichas fuentes provenían de sitios académicos reconocidos e indexados como: Google Académico, revistas científicas, tesis de posgrado, bases de datos como EBSCO y portales de revistas de universidades. Las referencias bibliográficas fueron recuperadas sobre todo en español, francés y portugués.

La metodología utilizada se orientó por la triangulación teórica de la información aportada por la teoría de Hernández et al. (2010), dicha triangulación aporta una diversidad de fuentes de datos, de investigadores, de teorías y de métodos, al contrastar unos con otros, para recolectar, analizar, confirmar e interpretar los datos. Lo que busca es identificar diferentes perspectivas para examinar el conjunto de la información. De acuerdo con Hernández et al. (2010), la investigación resulta más enriquecedora y profunda si la información proviene de distintos actores del proceso en indagación y si se obtiene a través de distintas técnicas. 
El procesamiento y análisis de la información recabada en la investigación se llevó a cabo a través de las siguientes etapas:

La primera etapa del análisis de la información fue la revisión de toda la información recabada mediante las distintas técnicas. Una segunda etapa fue la categorización y la codificación, lo anterior consiste en identificar y clasificar la información en unidades de análisis correspondiente a un texto, tópico o concepto, para darle sentido, esto se hará de acuerdo a las categorías de análisis establecidas para esta pesquisa.

La tercera etapa consistió en la tabulación de los datos para organizarlos mediante el proceso de categorización y codificación, a través de tablas que faciltaron el ordenamiento de la información y su visualización. Se realizó con tablas generales de categorías de las cuales se pueden desprender las subcategorías, de citas u otras. En este estadio de la investigación se hizo uso de la herramienta Atlas.ti, la cual posee un alto grado de versatilidad y facilitó la generación de las categorías de análisis mediante la construcción de conexiones entre los datos aportados por los participantes.

La cuarta etapa fue el procesamiento e implicó llevar a cabo todas las operaciones necesarias para relacionar los datos, encontrarles sentido y llegar a la interpretación. En la Tabla 1 se muestra la distribución de los participantes de la investigación.

Tabla 1.

Datos generales del estudiantado

\begin{tabular}{|c|c|c|c|c|c|}
\hline Participantes & Edad & País & Tiempo en CR & Últimos estudios realizados & Institución \\
\hline Est 1 & 30 & El Salvador & 1 año y 2 meses & $\begin{array}{l}\text { Administración de Empresas } \\
\text { (Carrera universitaria } \\
\text { incompleta) }\end{array}$ & \\
\hline Est 2 & 24 & Panamá & 1 año & $\begin{array}{l}\text { Ingeniería Civil } \\
\text { (Carrera universitaria } \\
\text { incompleta) }\end{array}$ & $\begin{array}{l}\text { Universidad } \\
\text { Tecnológica de } \\
\text { Panamá }\end{array}$ \\
\hline Est 3 & 32 & Haití & 2 meses & Colegio & Blaise Pascal \\
\hline Est 4 & 22 & Nicaragua & 3 años & Colegio & \\
\hline Est 5 & 26 & Haití & 2 años & Colegio & \\
\hline Est 6 & 26 & Costa Rica & & $\begin{array}{l}\text { Ciencias Políticas } \\
\text { (Carrera universitaria } \\
\text { completa) }\end{array}$ & $\begin{array}{l}\text { Universidad de } \\
\text { Costa Rica }\end{array}$ \\
\hline Est 7 & 22 & Costa Rica & & Colegio & \\
\hline Est 8 & 26 & Colombia & 1 año y 2 meses & $\begin{array}{l}\text { Licenciatura en Filosofia/ } \\
\text { ERE (Carrera universitaria } \\
\text { incompleta) }\end{array}$ & $\begin{array}{l}\text { Universidad de } \\
\text { Santo Tomás }\end{array}$ \\
\hline
\end{tabular}




\begin{tabular}{|c|c|c|c|c|c|}
\hline Participantes & Edad & País & Tiempo en CR & Últimos estudios realizados & Institución \\
\hline Est 9 & 35 & El Salvador & 1 año y 2 meses & Colegio & $\begin{array}{l}\text { Instituto } \\
\text { Nacional de } \\
\text { Chalchuapa }\end{array}$ \\
\hline Est 10 & 28 & Panamá & 1 año y 3 meses & $\begin{array}{l}\text { Bellas Artes } \\
\text { (Carrera universitaria } \\
\text { completa) }\end{array}$ & $\begin{array}{l}\text { Universidad de } \\
\text { Panamá }\end{array}$ \\
\hline Est 11 & 26 & Nicaragua & 1 año y 6 meses & $\begin{array}{l}\text { Administración de empresas } \\
\text { (Carrera para-universitaria } \\
\text { completa) }\end{array}$ & UENIC \\
\hline Est 12 & 19 & El Salvador & 1 año y 2 meses & Colegio & \\
\hline Est 13 & 25 & Haití & 1 año y 9 meses & $\begin{array}{l}\text { Técnicas de computación } \\
\text { (Carrera universitaria } \\
\text { incompleta) }\end{array}$ & UNIQUA \\
\hline Est 14 & 30 & Honduras & 1 año y 2 meses & $\begin{array}{l}\text { Universidad (Carrera } \\
\text { universitaria incompleta) }\end{array}$ & $\begin{array}{l}\text { Universidad } \\
\text { Tecnológica de } \\
\text { Honduras } \\
\end{array}$ \\
\hline Est 15 & 27 & Honduras & 1 año y 2 meses & $\begin{array}{l}\text { Administración de empresas } \\
\text { (Carrera universitaria } \\
\text { incompleta) }\end{array}$ & UNAH \\
\hline Est 16 & 19 & Honduras & 1 año y 2 meses & Colegio & \\
\hline Est 17 & 19 & México & 1 año y 2 meses & Colegio & \\
\hline Est 18 & 32 & El Salvador & 1 año y 2 meses & Colegio & \\
\hline
\end{tabular}

Nota: Elaboración propia de la autora.

Tabla 2.

Datos generales del profesorado

\begin{tabular}{|c|c|c|c|c|c|}
\hline Entrevistado & Sexo & Edad & País de origen & $\begin{array}{c}\text { Lugar de } \\
\text { residencia }\end{array}$ & $\begin{array}{c}\text { Horas por semana } \\
\text { dedicadas a la } \\
\text { docencia }\end{array}$ \\
\hline 1 & M & 36 & Costa Rica & Cartago & 20 \\
\hline 2 & F & 30 & Costa Rica & Cartago & 40 \\
\hline 3 & F & 30 & Costa Rica & San José & 20 \\
\hline 4 & M & 33 & Costa Rica & San José & 30 \\
\hline 5 & F & 28 & Costa Rica & San José & 20 \\
\hline 6 & M & 33 & Costa Rica & San José & 20 \\
\hline 7 & F & 45 & Costa Rica & San José & 10 \\
\hline
\end{tabular}

Nota: Elaboración propia de la autora.

\section{Resultados}

En este apartado, se presentan los principales factores que dificultan el aprendizaje de la filosofía en el estudiantado de la Universidad 
Teológica de América Central (UTAC) desde dos perspectivas: la docente y la discente.

\section{A) Perspectiva docente}

\section{A.1) Factores culturales}

Tres de siete docentes (N. ${ }^{\circ}$, N. ${ }^{\circ} 5$ y N. $\left.{ }^{\circ} 7\right)$ indican que un elemento que obstaculiza el aprendizaje de la filosofía es el factor cultural. Según la docente N. ${ }^{\circ} 5$, en un primer momento se da un choque cultural del país del que se procede al que se ingresa. Además, de "un aislamiento social" que tienen desde que llegan y hasta que se van, residen en el país, pero no lo conocen. Lo anterior genera una coartación en el aprendizaje, "que se refleja en la capacidad de retención y atención de las lecciones".

Luego, indica el profesor N. ${ }^{\circ} 2$ que al estudiante extranjero "se le dificulta incorporarse al sistema educativo costarricense por cuestiones culturales". La enseñante $\mathrm{N} .^{\circ} 7$ afirma que el factor separación influye mucho, así como venir a un país que no conocen y sin familia. Algunos estudiantes nunca habían ido a la universidad, eso implica mucho trabajo formativo del proceso en el que se encontraban, pues al mismo tiempo estaban clarificando un proyecto de vida.

El factor del contexto geográfico es señalado por los docentes N. ${ }^{\circ} 2$ y N. ${ }^{\circ} 6$, ambos coinciden en que "al estudiante extranjero le toma más tiempo habituarse" al sistema educativo de Costa Rica por razones contextuales y del entorno de procedencia.

Asimismo, los sistemas educativos de procedencia poseen diferentes niveles de exigencia, de ahí que la profesora N. ${ }^{\circ} 5$ manifiesta que otro factor que incide es la rigidez del sistema educativo de procedencia, ya que depende del país varía la "gradualidad" de exigencia de los cursos que han llevado.

La docente N. ${ }^{\circ} 7$ manifiesta que el factor cultural está asociado con que no sean "muy asiduos al uso de la biblioteca". También señala que, con los estudiantes de la Universidad Teológica de América Central, sí había posibilidades de hablar sobre su proyecto de vida como una decisión en la que debían ir trazando metas para lograrla. Detalla que son "grupos cautivos" que hay que ir conociendo y que están en una situación de vida muy específica, ellos viven en comunidades, en las que celebran y conviven con otros, están readaptándose. Aunado a lo anterior está el factor socioafectivo, ya que "ellos también están 
dudando y replanteándose su proyecto de vida, eso afecta el aprendizaje de la filosofía".

Los profesores N. ${ }^{\circ} 2$ y N. ${ }^{\circ} 4$ concuerdan en que otro factor que influye en el aprendizaje de la filosofía es la distancia de su país, el desprendimiento de la familia, las amistades y las costumbres propias de su tierra natal.

Es importante señalar que la filosofía en varios países de América Latina no forma parte de las cuatro materias principales del currículo oficial de enseñanza secundaria, algunos estudiantes la asumen como una materia sin importancia o extracurricular, debido a lo anterior la enseñante $N .^{\circ} 3$ considera que los discentes ingresan a la Universidad Teológica de América Central "con poco o casi nulo conocimiento de la filosofía, e interés por la misma", debido a que en los países de procedencia de los muchachos no se le da mucha importancia a la disciplina filosófica.

Cinco de los siete docentes (N. ${ }^{\circ} 1, \mathrm{~N} .{ }^{\circ} 2, \mathrm{~N}^{\circ}{ }^{\circ}, \mathrm{N} .^{\circ} 6$ y N..$\left.^{\circ} 7\right)$ coinciden en que el factor idioma es relevante en los estudiantes haitianos, toda vez que condiciona el aprendizaje, ya que al llegar a Costa Rica "no manejan la lengua española", lo que limita la lectura y la escritura. La profesora N. ${ }^{\circ} 7$ aclara que le ha sucedido lo mismo con estudiantes africanos, garífunos y guatemaltecos de origen indígena.

\section{A.2) Factores de comprensión lectora deficiente}

En general, los docentes reconocen que existen problemas de comprensión lectora deficiente. Esto se debe a falencias en la educación primaria y secundaria propia de los discentes. Según el docente N. ${ }^{\circ} 1$, no siempre se puede generalizar pues ha tenido estudiantes de Guatemala y Nicaragua "sobresalientes", e inclusive considera que "las deficiencias son más marcadas en los discentes de Haití por la cuestión del idioma. Sin embargo, la docente N. ${ }^{\circ} 7$ considera que las limitantes de estos últimos son comparables a las de los costarricenses que provienen de zonas rurales o alejadas del Valle Central. Reitera que la "pertenencia a ciertas congregaciones es un mejor predictor" de la formación de los educandos, sobresalen los dominicos, conventuales y capuchinos.

Los docentes N. ${ }^{\circ} 2, \mathrm{~N}^{\circ}{ }^{\circ} 3$ y N. ${ }^{\circ} 7$ coinciden en que esta deficiencia se asocia con problemas de lectura. En comparación con el costarricense, "el extranjero presenta más problemas para hacer una lectura adecuada y hacer un uso adecuado de las fuentes de información, 
redacción y formulación de argumentos". Además consideran que los estudiantes extranjeros, enfáticamente los centroamericanos y haitianos "tienen niveles de lecto-escritura y razonamiento lógico bajo".

La educadora $\mathrm{N}^{\circ}{ }^{\circ} 7$ señala que la incomprensión de los textos obedece a un "asunto afectivo" ya que el estudiante debe hacer un esfuerzo "por descentrar los contextos de los libros y su contenido". Otro elemento que agrava la deficiencia de la comprensión lectora es que los estudiantes que ingresan a la Universidad Teológica de América Central "no son asiduos a la lectura ni al uso de la biblioteca" o fuentes de información.

La docente N. ${ }^{\circ} 7$ explica que la comprensión lectora es deficiente en el sentido de que "los alumnos refieren niveles de comprensión muy literales, menos inferenciales y críticos, no es que los costarricenses sean mejores pero alcanzaban a obtener un nivel inferencial". El "nivel crítico se pudo observar en los discentes que ya venían con algunos estudios universitarios". La profesora ha observado que la comprensión lectora aumenta conforme crece la cantidad de materias cursadas y las exigencias.

Durante las entrevistas a profundidad los docentes coincidieron en que hay problemas de análisis y de síntesis. En este último rubro hay discentes que no alcanzan a sintetizar la idea principal de un texto tanto en el nivel escrito como en el nivel oral. En lo referente al análisis sucede que "no alcanzan a distinguir ideas secundarias de principales", ni a ordenar el argumento del documento de una forma coherente. Lo anterior se refleja en problemas de expresión oral y escrita.

Los docentes N. ${ }^{\circ} 1, \mathrm{~N} .^{\circ} 2, \mathrm{~N}^{\circ}{ }^{\circ} 3, \mathrm{~N} .^{\circ} 6$ y N. ${ }^{\circ} 7$ coinciden en que el problema de comprensión lectora deficiente, en el caso de los estudiantes haitianos, se deriva del factor del idioma, dado que los estudiantes no manejan el español al ingresar a la Universidad Teológica de América Central. Lo anterior remite a problemas de expresión oral, pues "no están habituados a realizar presentaciones orales con orden en las ideas, articulación y buena pronunciación".

La docente N. 5 anota que el problema de comprensión lectora deficiente se asocia también con que los estudiantes muestran un "escaso banco de vocabulario" al momento de expresarse oralmente y por escrito.

La académica $\mathrm{N}^{\circ} 7$ indica que el problema de comprensión lectora deficiente se relaciona con que los estudiantes "no conocen el sistema lingüístico de la filosofía, debido a que falta mucho contacto con la literatura pues casi no leen". Ese contacto les permitiría familiarizarse con los textos y el sistema lingüístico de esta disciplina. 
Los enseñantes N. ${ }^{\circ} 1, \mathrm{~N} .^{\circ} 3, \mathrm{~N} .^{\circ} 5, \mathrm{~N} .^{\circ} 6$ y N. ${ }^{\circ} 7$ concuerdan en que el problema de comprensión lectora deficiente se liga a problemas de expresión escrita, esto porque el estudiante "escribe con dificultad, sin reformular las ideas o es simple repetición, además con graves errores de ortografía".

De acuerdo con la docente N. ${ }^{\circ} 7$, los alumnos que "no cuentan con las adecuadas herramientas" para la comprensión lectora y la escritura recurren al plagio o al copypage de algunos textos. Considera ella que no con mala intención sino por la urgencia de resolver una necesidad inmediata.

\section{A.3) Factores para la pérdida de cursos}

Este factor se puede comprender desde dos núcleos, el primero atañe los aspectos humanos y el segundo el exceso de actividades extrauniversitarias u extracurriculares. Los docentes N. ${ }^{\circ} 2$ y N. ${ }^{\circ} 7$ coinciden en que es importante comprender ciertos aspectos humanos, como que el estudiante extranjero al ingresar a Costa Rica se desprende de su contexto espacio-temporal y psicosocial, de su familia, de sus amigos y todo lo que conoce de su lugar de origen, algunos nunca habían ido a la universidad. Se dan muchos elementos de separación.

Los profesores N. ${ }^{\circ} 1, \mathrm{~N} .{ }^{\circ} 2$ y N..$^{\circ} 3$ consideran que hay estudiantes que se "desentienden de su compromiso con el estudio". Esto puede obedecer a motivos personales, alguna situación en clase o dentro de la congregación en la que se encuentran. El profesor N ${ }^{\circ} 3$ reitera que "existe mucha falta de compromiso por parte de los estudiantes".

Los docentes N. ${ }^{\circ} 1, \mathrm{~N} .{ }^{\circ} 2$ y N. ${ }^{\circ} 4$ conciertan que el exceso de actividades extracurriculares es uno de los factores que incide para la pérdida de los cursos. Y esto se debe a que la vida religiosa en la que están inscritos los estudiantes les demanda muchas responsabilidades, que no siempre están bien organizadas y sopesadas con su experiencia de vida. Por tanto, los enseñantes N. ${ }^{\circ} 1, \mathrm{~N} .{ }^{\circ} 2, \mathrm{~N} .{ }^{\circ} 6$ y N. ${ }^{\circ} 7$ concuerdan que "existe mucha irresponsabilidad estudiantil", varios estudiantes no entregan los trabajos, otros entregan solo algunas asignaciones, pero pierden puntos de otras. Lo anterior, debido a las extenuantes jornadas de trabajo en las casas de formación y mal distribuidas en el tiempo según le han indicado los mismos discentes al profesorado. Por su parte los formadores, consideran que se debe más a errores en la organización del tiempo por parte del estudiantado, pues todos tienen asignados horarios de estudio. 
Los exámenes son parte de la evaluación normal en una universidad, sin embargo, los discentes no siempre toman conciencia del valor porcentual asignados a los mismos, frente a eso los profesores N. ${ }^{0} 1 \mathrm{y}$ N. ${ }^{\circ} 6$ convienen en que algunos estudiantes no recuperan las notas bajas, pues pierden porcentajes altos en los exámenes.

El docente N. ${ }^{\circ} 1$ expresa que el exceso de actividades extrauniversitarias incide en el bajo rendimiento de los alumnos. Finalmente, los educadores N. ${ }^{\circ} 1$ y N. ${ }^{\circ} 6$ enfatizan que los estudiantes desertan del curso lectivo debido a decisiones internas de las congregaciones religiosas y personales. Las autoridades universitarias "nunca informan al docente", este se percata porque los otros alumnos le expresan que el compañero se fue. Otros han dejado el curso.

\section{B) Perspectiva estudiantil}

\section{B.1) Factores por los sistemas educativos de procedencia}

\section{Estudiantes de EI Salvador}

Los discentes salvadoreños indican que los factores que dificultan su aprendizaje de la filosofía emanan de la formación que brinda el sistema de educación salvadoreño y las deficiencias que se arrastran de secundaria. De acuerdo con el estudiante N. ${ }^{\circ} 18$, se brinda una formación que es "sólo para salir del paso"; es decir, se educa en lo básico, para saber leer, escribir y sumar. Aunado a lo anterior, los alumnos de El Salvador presentan "una gran falta de criticidad", debido a que al estudiante salvadoreño "se le enseña a pensar hasta que llega a la universidad". Expresan que parte de sus dificultades en el aprendizaje de la filosofía reside en que él tiene problemas para analizar y sintetizar, esto debido a la formación en su país de procedencia. Además él aduce que no tiene la misma capacidad ni formación que los costarricenses, por el tiempo en que ha estado en este país ha constatado las diferencias de formación y "concluye que los estudiantes costarricenses tienen una mejor preparación educativa".

El discente N. ${ }^{\circ} 12$ señala que un factor que le dificulta el aprendizaje de la filosofía reside en el cambio de evaluación, pues estaba acostumbrado a exámenes con opciones de respuesta para escoger, afirma que "no tenía nada que pensar". Pero cuando llega a la universidad en Costa Rica le realizan exámenes en los que le piden desarrollo y nunca había hecho ese ejercicio. Otro factor fue "el hecho de tener que 
pensar, pues en El Salvador no le enseñaron ni analizar, ni a deducir, ni a razonar o a aplicar lo aprendido".

Como estudiante, "no está habituado a la lectura de un libro completo", considera que en los cursos de filosofía de primer año de la Universidad Teológica de América Central no se debe solicitar al estudiante este tipo de ejercicios.

\section{Estudiantes de Panamá}

El estudiante panameño N. ${ }^{\circ} 2$ indica que hay compañeros que vienen con deficiencias de secundaria y por ende con desniveles académicos. "Los docentes por querer nivelarlos a todos afectan a los que pueden aprender más rápido". Esto hace que se pierda el interés en el conocimiento.

\section{Estudiantes de Nicaragua}

Los estudiantes nicaragüenses N. ${ }^{\circ} 4$ y N. ${ }^{\circ} 11$ detallan que parte de los factores que les dificultan el aprendizaje de la filosofía se derivan de un nodo principal que son las deficiencias que arrastran desde la educación secundaria.

El estudiante N. ${ }^{\circ} 4$ expone que tres factores que obstaculizaron su aprendizaje de la filosofía "fue su falta de abstracción, su incapacidad de análisis y que nunca desarrolló habilidades de comprensión lectora", todo lo anterior derivado de las deficiencias en su educación secundaria.

El alumno N. ${ }^{\circ} 11$ establece que cuando ingresó a la universidad presentaba desfases en el aprendizaje del inglés y de la estadística. Debido a las deficiencias en secundaria "él ingresa con una serie de habilidades no desarrolladas por lo que genera mucha frustración observar que había compañeros que avanzaban más rápido en ciertos conocimientos que él aún no dominaba".

\section{Estudiantes de Honduras}

El estudiante hondureño N. ${ }^{\circ} 16$ menciona que "él es incapaz de llevar todas las materias al día por el exceso de carga académica". A él le gusta aprender, pero con tanta carga de materias no disfruta las clases, pues es mucha la presión que tiene.

El compañero N. ${ }^{\circ} 15$ aduce que "es incapaz de poder seguir a los docentes que construyen esquemas en la pizarra, y que le molesta el timbre de voz de algunos. Además reconoce que no tiene hábitos de lectura para llevar el ritmo de todo lo que hay que leer". 


\section{Estudiante de México}

El discente N. ${ }^{\circ} 2$ señala que "hay estudiantes que vienen desfasados y con malas bases por sus sistemas educativos" y por eso les costó el curso de Introducción a la lógica.

\section{B.2) Factores pedagógicos}

\section{Estudiantes de El Salvador}

El discente N. ${ }^{\circ} 18$ expone que al ingresar a la universidad "en clases largas de tres horas o más él tiene dificultad para concentrarse". También le era difícil fijar los conocimientos, porque él aprende de manera interactiva o lúdica.

El alumno N. ${ }^{\circ} 12$ considera que el cambio de evaluación fue un factor que dificultó su aprendizaje de la filosofía, pues "debía analizar y desarrollar argumentos, algo a lo que no estaba acostumbrado, pues siempre le aplicaron pruebas de selección no de razonamiento".

\section{Estudiantes de Panamá}

El alumno N. ${ }^{\circ} 10$ considera que un factor que dificulta el aprendizaje de la filosofía es que los profesores en los cursos quieren atiborrar al estudiante de mucho material que no tiene tiempo de leer.

El discente N. ${ }^{\circ} 2$ explica que otro elemento que dificulta el aprendizaje de la filosofía es que al inicio los cursos son muy memorísticos, considera que al principio es correcto, más conforme pasa el curso se puede avanzar a otro nivel de pensamiento.

\section{Estudiantes de Nicaragua}

El estudiante N. ${ }^{\circ} 11$ anotó que a su ingreso a la universidad le costó adaptarse a los profesores. Además de que en la universidad las clases son más exigentes.

\section{Estudiantes de Honduras}

El estudiante N. ${ }^{\circ} 15$ indica que un factor que dificultó su aprendizaje de la filosofía se da cuando le han dado clases docentes sin mucha capacidad correctiva.

\section{Estudiante de México}

El estudiante N. ${ }^{\circ} 17$ precisó que una de las dificultades es la intención de querer abarcar mucha materia, pero no sabe si se debe a que los docentes deben cumplir un programa. Otro aspecto es que "los 
docentes abren espacios para que los estudiantes participen, pero ellos no lo hacen, les falta iniciativa".

\section{Estudiante de Colombia}

El discente $\mathrm{N} .{ }^{\circ} 8$ señala que una dificultad para el aprendizaje de la filosofía se deriva de cuando "la clase se reduce solo a exposiciones de los compañeros, de modo que no se le saca provecho al aprendizaje en clase".

\section{Estudiantes de Costa Rica}

El estudiante N. ${ }^{\circ} 7$ afirma que se le dificulta el aprendizaje de la filosofía cuando los docentes pasan de un tema a otro sin orden cronológico de la información.

\section{B.3) Factores personales}

\section{Estudiantes de EI Salvador}

Indica el alumno N. ${ }^{\circ} 18$ que sus dificultades radicaron en "la falta de madurez emocional, así como la falta de sensibilidad por parte de los estudiantes y profesores que le planteaban un modo ideal de ser de la filosofía".

\section{Estudiantes de Panamá}

Los estudiantes panameños apuntan como factor que dificulta el aprendizaje "la falta de madurez de los estudiantes que ingresan a la Universidad Teológica de América Central. Además de la disposición de los estudiantes para aprender y la falta de hábitos de lectura para un buen aprendizaje".

\section{Estudiantes de Nicaragua}

Precisan que los factores que dificultaron su aprendizaje han sido "la pérdida de los amigos y la adaptación a los nuevos profesores".

\section{Estudiantes de Honduras}

Los estudiantes hondureños coinciden en que el factor que más dificulta el aprendizaje de la filosofía es el exceso de carga académica que les exige el formador de la orden religiosa que deben llevar. Debido a eso los tres discentes afirman que siempre "están cansados y estresados". Lo anterior provoca que también "lean de manera superficial o por encima. Aunado a lo anterior no asimilan con responsabilidad el material de los cursos, pues están cargados con tanta materia que no siempre lo pueden hacer de la mejor forma". Un último aspecto es "la 
no preparación de la clase por parte del alumno", ya que la responsabilidad es conjunta con el profesor, pero por el exceso de actividades, ellos "no dan abasto con todas las responsabilidades".

\section{Estudiantes de Costa Rica}

Los alumnos costarricenses coinciden en que el estudiante de la Universidad Teológica de América Central "no es responsable con sus labores como la de realizar sus lecturas".

\section{Estudiantes de Haití}

Para los estudiantes haitianos el factor que les dificultó el aprendizaje de la filosofía fue el idioma.

\section{Discusión de los resultados}

Respecto a las dificultades por sistema educativo de procedencia se pudo constatar que en general los estudiantes salvadoreños, los panameños, los nicaragüenses, los hondureños y el mexicano coincidieron en que uno de los factores que entorpecen el aprendizaje de la filosofía deriva de las falencias educativas que varios arrastran desde la secundaria. Aducen que en ciertos países como el Salvador se les enseña lo básico, es decir a leer y escribir, pero no se enseña a analizar, a razonar, o a sintetizar el conocimiento. Por su parte, los discentes nicaragüenses coinciden en que las deficiencias de secundaria no les permitieron desarrollar como corresponde habilidades de abstracción, de análisis y de comprensión lectora, esto último también compartido por los alumnos hondureños quienes van más allá y reconocen que no tienen hábitos de lectura. Lo anterior ha sido un condicionante para llevar un adecuado ritmo de lectura como se demanda en el nivel universitario.

Propiamente los estudiantes de El Salvador aquejan que su sistema educativo los prepara solo en lo básico para saber leer, escribir y sumar, al mismo tiempo hay una falta de criticidad y el sistema educativo no les enseña a pensar hasta que ingresan a la universidad, llegan a la educación superior con insuficientes conocimientos para realizar ejercicios de análisis y síntesis.

Asimismo, señala el educando N. ${ }^{\circ} 12$ que otro factor que no favorece el aprendizaje de la filosofía reside en que no está habituado a la lectura de libros completos y que esto no se debería hacer en el primer año de la universidad. Al respecto, los estudios de Velásquez (2002) han comprobado que los estudiantes latinoamericanos leen en promedio un 
libro al año, mientras que los de países desarrollados más de quince. El problema radica en que la lectura para los discentes universitarios es vista como un tedio, la falta de interés según Calderón y Quijano (2010) está desde mucho antes de ingresar a la educación superior. Este estudiante salvadoreño pone sobre la mesa un problema que no está presente solo en su país, sino en varios países de América Latina y el mundo, la lectura no se visualiza como una cultura, ni se le asigna mayor relevancia, aun cuando los postulados de la Organización para la Cooperación y Desarrollo Económico (OCDE) señalan que esta es vital, toda vez que promueve el desarrollo de procesos cognitivos como: comprender, analizar, sintetizar, criticar y reelaborar nuevos saberes.

Un tercer factor que obstaculiza el aprendizaje de la filosofía estriba para este estudiante salvadoreño en que no estaba habituado a ejercicios de desarrollo en los que tuviera que razonar, pues en su país la evaluación consistía en escoger las respuestas, no tenía que pensar como él lo indica. Esta situación es alarmante ya que significa que no se están fomentando en los alumnos habilidades para ser autónomos y pensar por ellos mismos. Espitaleta (1993) anota que el problema de los estudiantes que llegan a la universidad a estudiar filosofía es que no tienen los instrumentos necesarios para pensar y reflexionar, pero agregado a eso no se les ha fomentado una disposición al análisis, a la síntesis, a la emisión de juicios o de opiniones y, menos aún, a la lectu$\mathrm{ra}$, a la escritura y sus implicaciones.

El discente N. ${ }^{\circ} 4$ de Nicaragua apunta que los tres principales factores que dificultaron su aprendizaje de la filosofía fueron su falta de abstracción, su incapacidad de análisis y que nunca le desarrollaron habilidades de comprensión lectora, deficiencias similares a las de su compañero de El Salvador. Ante esta situación la UNESCO (2009) advierte que en general los estudiantes que ingresan por primera vez a estudiar filosofía la desconocen, luego presentan un manejo deficiente de las abstracciones, para Espitaleta (1993) también hay que incluir dificultades con la lógica, el raciocinio e inferencias. Sin embargo, lo precedente se puede comprender más holísticamente desde los postulados de Rojas y Jiménez (2012) quienes consideran que los estudiantes tienen problemas para realizar operaciones de análisis, síntesis y abstracción debido a una deficiente comprensión lectora, ya que esta provee los insumos necesarios para desarrollar estas habilidades de pensamiento. 
En lo atinente a los factores pedagógicos que dificultan el aprendizaje de la filosofía, se puede afirmar que los discentes en general no coincidieron, sino que se expresaron desde lo que particularmente les afecta a ellos como la pérdida de la concentración durante largas horas de clase para el salvadoreño $\mathrm{N}^{\circ}{ }^{\circ} 18$, pero también compartido con el estrés y el cansancio de los estudiantes hondureños $\mathrm{N} .^{\circ} 14, \mathrm{~N}^{\circ} 15$ y N. ${ }^{\circ}$ 16. Desde la perspectiva de los estudios realizados por Dina Krauskopf (2000) la falta de concentración, en el ámbito académico, se debe con frecuencia a la fatiga.

Los discentes panameños rescataron que los docentes los recargan con exceso de material, los estudiantes salvadoreños y nicaragüenses agregan que también con exceso de reportes y tareas. Dichas prácticas no son correctas ya que desde las teorías de Rengifo y Pinillo (2013) ha sido un error creer que la masiva imposición de obligaciones académicas garantiza un proceso óptimo de aprendizaje.

El alumno mexicano precisó el problema de querer abarcar mucha materia y la no participación del estudiante en clase. En cuanto al primer punto subraya Echeverría (2004) que la teoría del aprendizaje significativo reta a los docentes a plantear clases no necesariamente de muchas horas ni en exceso cargadas de contenido, pero sí con estructuras claras y productivas que promuevan en el aula espacios de comprensión genuina, en los que el educando tenga más libertad de expresarse y construir sus propias ideas. Acerca del segundo aspecto Espitaleta (1993) suscribe que los alumnos y las alumnas tienen la oportunidad de preguntar y participar, pero no lo hacen porque se sienten más cómodos al tomar nota y escuchar a un profesor en la clase magistral. Por su parte Gutiérrez y Montes de Oca (2007) alegan que algunas razones para la no participación del alumno están en que no lee o no domina los contenidos estudiados.

Ciertamente, el exceso de actividades extracurriculares sí interviene en el aprendizaje de la filosofía debido a que según Gutiérrez y Montes de Oca (2007) un estudiante universitario debe dedicar al menos trece horas por semana solo para la lectura, y estos estudiantes declaran que no cuentan con el tiempo suficiente para efectuar ni la lectura ni los procesos de apropiación de los conocimientos. Agrega Difabio (2015) que el principal obstáculo para adquirir los aprendizajes, es que estos no están siendo interiorizados. 
Respecto al factor tiempo Krauskopf (2000) precisa que algunas escogencias de vida imponen horarios rígidos y normativos que postergan las necesidades de recreación, lo que torna el estudio como una actividad privativa y obligada. La opción por la vida religiosa es una escogencia de vida que tiene sus propias exigencias.

En el caso de los estudiantes haitianos la principal dificultad en el aprendizaje de la filosofía recae en el desconocimiento del español, lengua en la que se imparten las clases. De acuerdo con la UNESCO (2011),

La dificultad más pertinente es la lengua, puesto que para comprender los conceptos hay que comprender la lengua de enseñanza. A esto se añade el carácter específico del saber filosófico, con sus conceptos y la divergencia y la diversidad de las ideas. (p. 81)

La UNESCO (2011) señala tres consecuencias negativas de utilizar una lengua extranjera, que además no se domina al momento de iniciar la formación en filosofía y en otras áreas de conocimiento: primero existe una baja motivación, luego estos son mal comprendidos y tercero se disminuye la capacidad de análisis y de reflexión de los estudiantes. Lo anterior debido a que el lenguaje y el idioma determinan las categorías conceptuales con las que entendemos el mundo y accedemos al conocimiento.

Los factores personales que dificultan el aprendizaje de la filosofía para los salvadoreños, costarricenses, panameños y hondureños pasan por la inmadurez emocional para asumir el compromiso con el estudio, esto a su vez está ligado con la falta de hábitos de lectura, la responsabilidad para efectuar las lecturas y la disposición para aprender. Para los estudiantes costarricenses y hondureños el discente de la Universidad Teológica de América Central no asume con seriedad la preparación de la clase, deber que no recae exclusivamente en el profesor, ya que según Echeverría (2004) una de las metas del aprendizaje de la filosofía es que el alumno se entienda como un ente capaz, autónomo y sujeto activo de su proceso de enseñanza-aprendizaje.

No obstante, la falta de madurez emocional para asumir el estudio se puede comprender desde la teoría de Krauskopf (2000) quien explica como en la vida de todo estudiante hay reacciones de ajuste, el cambio de colegio a universidad es un momento propicio en el que "según las condiciones sociales y emocionales, así como sus experiencias 
variaran sus comportamientos, hasta que paulatinamente se consoliden sus patrones conductuales" (p. 97). Aunado a lo anterior la escogencia de un proyecto de vida lleva una serie de dudas y conflictos que solo con el tiempo se logran canalizar. Madurar la escogencia de la profesión es complejo, algunos no tienen claro si desean ir a la universidad o no, o qué carrera escoger. Lo único cierto para esta especialista en educación y adolescencia es que cuando el discente se encuentra en la carrera que desea, que se ajusta a sus expectativas y que se identifica con lo que quiere para su vida, hará un esfuerzo por comprometerse con el estudio para ser un profesional.

\section{Conclusiones}

Los factores que dificultan el aprendizaje de la filosofía para los estudiantes de la Universidad Teológica de América Central (UTAC) obedecen en gran medida a deficiencias en la formación secundaria de sus respectivos países que no incentivan hábitos de lectura, el desarrollo de la comprensión lectora, procesos de análisis y síntesis, así como espacios para la discusión de las ideas en las que se pueda estimular la participación activa, la criticidad y el aprendizaje.

Aunado a lo anterior no se puede subestimar los aspectos socio-afectivos que se derivan de la escogencia de un proyecto de vida en las órdenes religiosas, puesto que desde su ingreso a Costa Rica el alumno se confronta con una nueva realidad que desconocía, con una nueva cultura, sin amigos, sin familia e integrándose a nuevos procesos de convivencia con sus iguales. Para varios es la primera vez que salen de su país, que van a la universidad y que se introducen al mundo de la filosofía. La filosofía como disciplina demanda el dominio de ciertas habilidades que solo se desarrollan con la lectura, la discusión y comprensión de las ideas, estimulando así la autonomía del educando universitario en la construcción de su propio aprendizaje.

La universidad como nuevo espacio educativo establece exigencias y compromisos en el nivel académico para las cuales los discentes no estaban preparados por motivos personales, formativos y de madurez. La asunción de un proyecto de vida y de una formación profesional implicó una serie de demandas en lo correspondiente al estudio para las cuales no estaban del todo bien instruidos y esto, a su vez, les dificultó el aprendizaje de la filosofía. 
Es recomendable que la institución comience a implementar medidas para facilitar el este aprendizaje en el alumnado, entre ellas se sugiere las siguientes:

$\checkmark \quad$ Implementar una prueba diagnóstica que provea información sobre la formación educativa, los niveles de razonamiento, las habilidades adquiridas, problemas de aprendizaje, áreas de conocimiento fuertes y débiles, para potencializar las primeras y trabajar las segundas, etc.

$\checkmark \quad$ Impartir cursos intensivos de español para los estudiantes que no manejan el idioma.

$\checkmark \quad$ Considerar la carga académica real que pueden matricular los estudiantes tomando en cuenta la gran cantidad de actividades extra-universitarias que deben realizar por su formación religiosa.

$\checkmark \quad$ Proponer un curso de Comprensión lectora para desarrollar habilidades que sean de utilidad en el aprendizaje de la filosofía en el nivel universitario, ya que la filosofía como disciplina especializada posee claves de lenguaje específicas para el ingreso y la comprensión de los textos que solo se desarrollan con la comprensión lectora.

$\checkmark \quad$ Promover hábitos de lectura que faciliten la adquisición de vocabulario, el desarrollo de habilidades de comprensión, análisis, síntesis, pensamiento crítico e inferencial.

$\checkmark \quad$ Crear talleres de estudio de textos de filosofía, de escritura y de argumentación filosófica para mejorar tanto la comprensión como la expresión de esta disciplina.

Es importante que las propuestas anteriores se desarrollen de manera conjunta y coordinada entre la parte administrativa de la institución, las casas de formación, el cuerpo docente y el estudiantado, con el fin de mejorar los procesos de enseñanza-aprendizaje de la filosofía.

\section{Referencias}

Amador, K. y Alarcón, L. (2006). Propuesta metodológica para evaluar la comprensión lectora en estudiantes universitarios. Revista Graffylia, 6, 126-135. Recuperado de https://dialnet.unirioja.es/ servlet/articulo? codigo $=2290600$ 
Arrieta, B. y Meza, R. (2005). La comprensión lectora y la redacción en estudiantes universitarios. Revista Iberoamericana de Educación, 36 (13) 1-10. Recuperado de https://rieoei.org/RIE/article/ view/3565

Barrantes, E. (2013). Investigación un camino al conocimiento. Un enfoque cuantitativo y cualitativo. San José: Costa Rica: EUNED.

Calderón, A. y Quijano, J. (2010). Características de comprensión lectora en estudiantes universitarios. Revista Estudios Socio-Jurídicos, 12 (1), 37-364. Recuperado de http://www.redalyc.org/ pdf/733/73313677015.pdf

Carvajal, A. y García J. (2004). ¿Cómo perciben los estudiantes universitarios la enseñanza de la Filosofía, según sus experiencias en la Educación Diversificada Costarricense? Revista Actualidades Investigativas en Educación, 4 (1), 1-20. Recuperado de http:// revista.inie.ucr.ac.cr/uploads/tx_magazine/FILOSOFIA.pdf

Claux, M. y La Rosa, M. (2004). La comprensión lectora en el aula: una experiencia significativa. Perú: Pontificia Universidad Católica de Perú.

Okoudjou, P. C. (2007). Comment enseigner aujourd'hui la philosophie en Afrique? En: HOUNTONDJI, P. J. (dir.). La rationalité une ou plurielle? Dakar: CODESRIA.

Difabio, H. (2005). Competencias para la comprensión de textos y el pensamiento crítico en el nivel medio y universitario (Tesis de doctorado no publicada). Universidad Nacional de Cuyo, Argentina. Recuperado de http://bdigital.uncu.edu.ar/objetos_digitales/4624/difabiocompcomprtextnivuniv.pdf

Echeverría, E. (2004). Filosofia para niños. México: Aula Nueva SM.

Espitaleta, S. (1993). El método formulensayo en la enseñanza de la filosofía. Revista de Educación y Pedagogía, 10 y 11, 103-118. Recuperado de http://aprendeenlinea.udea.edu.co/revistas/index. php/revistaeyp/article/view/5703/5123

Guevara, M. (2014). Estrategias de Aprendizaje Cooperativo y Comprensión Lectora con textos filosóficos en Estudiantes de Filosofía de la Facultad de Educación de la Universidad Nacional Amazónica de Madre de Dios, año 2012 (Tesis de Maestría no publicada). Universidad Mayor de San Marcos, Perú. Recuperado de http://cybertesis.unmsm.edu.pe/bitstream/cybertesis/3957/1/ Guevara_dm.pdf 
Gutiérrez, A. y Montes de Oca, R. (2007). La importancia de la lectura y su problemática en el contexto educativo universitario. El caso de la Universidad Juárez Autónoma de Tabasco (México). Revista Iberoamericana de Educación, 34(3), 1-12. https://doi.org/ https://doi.org/10.35362/rie3433265

Gómez, J. (2011). Perspectivas de la enseñanza de la filosofía en Costa Rica: El caso de la Universidad Nacional. Revista Praxis, 67, 121-128 Recuperado de https://www.revistas.una.ac.cr/index. $\mathrm{php} /$ praxis/article/view/5052/4834

Hernández, R., Fernández, C. y Baptista, P. (2010). Metodología de la Investigación (5ta). México: Mc. Graw Hill.

Krauskopf, D. (2000). Adolescencia y Educación. San José, C. R.: EUNED.

Rengifo, J. y Pinillo, H. (2013). Las problemáticas del proceso de enseñanza aprendizaje de la filosofía en Colombia. REVIBE Revista Iberoamericana de Ciencias Sociales, 1, 1-22. Recuperado de http://www.revibecs.com/wr-resource/ent8/1/Las\%20problematicas $\% 20 \mathrm{del} \% 20$ proceso $\% 20 \mathrm{de} \% 20 \mathrm{ea} \% 20 \mathrm{de} \% 201 \mathrm{l} \% 20$ filosof\%C3\%ADa\%20en\%20Colombia.pdf

Rojas, G. y Jiménez, H. (2012). La educación superior desde la lectura y la escritura. Revista Amazonia Investiga, 1, 19-35. Recuperado de http://www.udla.edu.co/re-vistas/index.php/amazonia-investiga/ article/view/3

UNESCO. (2009). Enseñanza de la filosofía en América Latina y el Caribe. París, Francia.: Autor. Recuperado de http://unesdoc. unesco.org/images/0018/001851/185119s.pdf

UNESCO. (2011). La filosofía. Una escuela de la libertad. Enseñanza de la Filosofia y Aprendizaje del Filosofar: la situación actual y las perspectivas para el futuro. Paris, Francia: Autor. Recuperado de http://unesdoc.unesco.org/images/0019/001926/192689S.pdf

Velásquez, R. (2002). Dificultades para enseñar filosofía en una realidad escolar. Revista Cuestiones de Filosofia, 3-4, 1-7. Recuperado de http://revistas.uptc.edu.co/revistas/index.php/cuestiones_filosofia/ article/view/587/585

Vygotsky, L. (1995). Pensamiento y lenguaje. Cognición y desarrollo humano. España: Paidós. 